\title{
A moiré foray
}

\author{
The transport properties of many two-dimensional systems are strongly affected by the proximity of a periodic \\ pattern. Colloidal particles are now shown to have preferred sliding routes due to competing symmetries between
} two unmatched crystalline surfaces.

\section{Pietro Tierno}

$\Lambda$ s Newton first intuited, a particle subjected to an external force moves along the direction prescribed by this force unless contact with the medium or a nearby surface opposes its motion. But what happens if the surface creates a periodic potential, such as that generated by an array of atoms on a flat interface? In this situation, the particle can be forced to move along a periodic trajectory commensurate with the lattice, giving rise to a 'Devil's staircase' comprising characteristic stair-like plateaus in the migration angle. This effect - known as directional locking - has been observed in many different contexts, from the pinning of electrons ${ }^{1}$ or vortices in high-temperature superconductors ${ }^{2}$, to colloids in optical lattices ${ }^{3}$ and flowing microfluidic streams ${ }^{4}$. Now, writing in Nature Physics, Xin Cao and colleagues have observed locking effects in clusters of colloids, driven by gravity across a periodic array of microscopic holes ${ }^{5}$. The work shows that studies on friction, traditionally relegated to the macro- or nanoscale world, can also be approached with microscopic particles.

The array was fabricated via soft lithography to form a triangular lattice of wells of $4 \mu \mathrm{m}$ diameter and $80 \mathrm{~nm}$ depth. The pattern was then tilted by $15-20^{\circ}$ so that the colloidal particles dispersed in water slid across the substrate, floating a few nanometres above it. Instead of focusing on the motion of single particles, the authors placed large clusters of permanently linked paramagnetic colloids on the substrate, examining particles with different sizes and a lattice constant slightly smaller than the underlying pattern. Once in motion, the clusters displayed a complex dynamics rotating and translating via specific routes that did not coincide with the axis of the substrate. Their behaviour called for a deeper interpretation based on the presence of competing lattice symmetries.

As the clusters slid, they tried to minimize frictional forces by forcing the particles to accommodate optimal positions, namely by filling the maximum number of holes. But the substrate and the

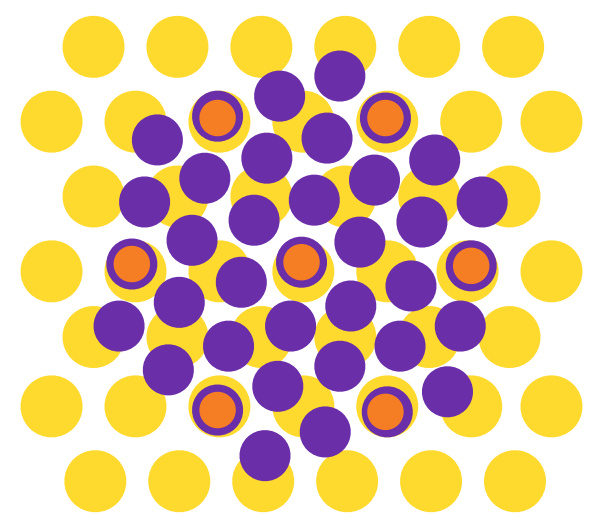

Fig. 1 | A colloidal cluster on top of a periodic substrate forms a moiré superstructure. Figure adapted from ref. ${ }^{5}$, Springer Nature Limited.

clusters were not identical, and mismatch between the two produced translational and angular rearrangements, resulting in a discontinuous, step-like behaviour of the position and orientation as a function of time. When superimposed at certain positions, the colloidal lattice and the underlying periodic substrate formed a moiré pattern (Fig. 1) - an interference pattern that arises when two similar but not completely identical structures are rotated or displaced with respect to one another. The complex route chosen by the clusters followed a sequence of stick-and-slip events whereby the moiré superstructure was periodically reformed as the clusters moved through particular lattice locations. Energy calculations and numerical simulations confirmed that, effectively, forming such a superstructure gives rise to an easy energetic path for the cluster to follow.

A related work from the same group previously revealed 'kink' and 'anti-kink' effects in colloidal crystals driven across optical landscapes ${ }^{6}$. At first glance, these colloidal systems may simply be considered toy models with which one can visualize ideal cases of perfectly symmetric sliding surfaces. But the studies ${ }^{5,6}$ also open new research directions beyond confirming atomistic models on friction, because different physical interactions come into play at colloidal length scales. For example, hydrodynamic interactions can become important, and fluid flow between two sliding surfaces may lead to reduced contact forces. This situation differs from that of an atomic force microscope tip driven above an atomically flat layer. By taking advantage of colloidal engineering, one could tune interactions with the substrate by reducing the cluster elevation, perhaps with a polymer depletant that screens electrostatics, or a magnetic field gradient, depending on the nature of the particles.

Cao and colleagues focused their work on stiff clusters with no internal motion. But mobile particles, such as those created by external fields ${ }^{7}$, may provide a way to visualize the deformation induced by the substrate and better understand how elastic stress develops during sliding. The competition between incommensurate structures in relative motion could also be explored on different geometries, as soft lithography offers the freedom to design any lattice geometry and pattern. Finally, the magnetic nature of the colloidal particles employed in this study make them responsive to external fields, a feature that the authors are yet to exploit - offering a welcome direction for future work.

\section{Pietro Tierno}

Department of Condensed Matter Physics, University of Barcelona, Barcelona, Spain. e-mail:ptierno@ub.edu

Published online: 13 May 2019 https://doi.org/10.1038/s41567-019-0541-5

References

1. Weiss, D. et al. Phys. Rev. Lett. 66, 2790-2793 (1991)

2. Reichhardt, C. \& Nori, F. Phys. Rev. Lett. 82, 414-417 (1999).

3. Korda, P. T., Taylor, M. B. \& Grier, D. G. Phys. Rev. Lett. 89 , 128301 (2002).

4. Huang, L. R., Cox, E. C., Austin, R. H. \& Sturm, J. C. Science 304, 987-990 (2004)

5. Cao, X., Panizon, E., Vanossi, A., Manini, N. \& Bechinger, C. Nat. Phys. https://doi.org/10.1038/s41567-019-0515-7 (2019).

6. Bohlein, T., Mikhael, J. \& Bechinger, C. Nat. Mater. 11, 126-130 (2012).

7. Martinez-Pedrero, F. \& Tierno, P. Phys. Rev. Appl. 3, 051003 (2015). 